\title{
Anisotropy in the Anodic Oxidation of Silicon in KOH Solution
}

\author{
Harold G. G. Philipsen* and John J. Kelly \\ Debye Institute, Utrecht University, P.O. Box 80000, 3508 TA, Utrecht, The Netherlands \\ Received: May 17, 2005; In Final Form: July 13, 2005
}

\begin{abstract}
The electrochemical oxidation and passivation of $\mathrm{Si}(100)$ and $\mathrm{Si}(111)$ electrodes in $\mathrm{KOH}$ solution was studied by potentiodynamic and potential-step measurements. Striking differences were observed between the surfaces. A comparison of the results for $\mathrm{n}$ - and $\mathrm{p}$-type electrodes led us to conclude that electrochemical oxidation of silicon in alkaline solution must be triggered by a chemical reaction. The strong influence of temperature on the current-potential and current-time results of (111) surfaces supports the importance of chemical activation. Photocurrent experiments on n-type (111) electrodes show that oxide nucleation is important for growth of the passive layer. A mechanism combining surface chemistry and electrochemistry is proposed to account for the pronounced anisotropy in anodic oxidation.
\end{abstract}

\section{Introduction}

The influence of crystallographic orientation on the kinetics of surface reactions is of fundamental interest and of practical importance in many systems. For example, chemical etching of silicon in alkaline solution is strongly anisotropic; the etch rate depends on the crystal face exposed to the etchant. ${ }^{1}$ This dependence allows a range of characteristic forms to be etched in single-crystal silicon, a process widely used in MEMS (microelectromechanical systems). ${ }^{2,3}$

Anodic oxidation of silicon is also an important step in MEMS technology. For example, an electrochemical etch stop based on the passivation of a reverse-biased $p-n$ junction is used in beam and membrane fabrication. ${ }^{4,5}$ While it is known that the anodic properties of silicon depend on crystallographic orientation, ${ }^{6,7}$ little attention has been devoted to the origin of this dependence. There are various reasons for considering anodic reactions at the different faces. Galvanic interaction between facets exposed during chemical etching of silicon determines the open-circuit potential and thus the etching anisotropy ${ }^{8}$ the electrochemical reactions occurring at the different facets are clearly important. Selective passivation can be used to control the relative etch rates of different faces in device fabrication. Anisotropy in anodic oxidation can serve as a diagnostic tool in micromachining. ${ }^{9}$ In addition, study of the electrochemistry of the anodic reactions might be expected to give information about the surface chemistry.

An unusual feature of the electrochemistry of silicon in alkaline solution is the strong similarity between the anodic current-potential characteristics of $\mathrm{n}$ - and p-type electrodes. ${ }^{10}$ This holds for both (100) and (111) surfaces. ${ }^{6,7}$ Anodic oxidation of most semiconductors involves a hole reaction via the valence band. ${ }^{11}$ Consequently, the rate is high for p-type but low for n-type semiconductors in the dark. Anodic oxidation of an n-type material can only be due to the injection of valence electrons from surface bonds into the conduction band. Direct thermal excitation of electrons from the valence band to the conduction band is energetically very unfavorable. In the case of silicon in alkaline solution, electron injection must occur from activated

* Corresponding author. Tel: +31-30-2532214. Fax: +31-30-2532403. E-mail: h.g.g.philipsen@phys.uu.nl. intermediates of the anisotropic chemical etching reaction. ${ }^{10,12-14}$ There is considerable evidence to show that for potentials before passivation occurs the anodic current of p-type $\mathrm{Si}(100)$ is also due to a large extent to an electron-injection process. ${ }^{12-16}$

Studies of the electrochemistry of silicon have been restricted almost exclusively to (100) and (111) electrodes. A comparison of these surfaces in alkaline solution reveals clear trends. For both types of electrode, the anodic current increases as the potential is scanned positive with respect to the open-circuit value $\left(U_{\mathrm{oc}}\right)$; in both cases, passivation occurs beyond a certain peak potential in the voltammogram $\left(U_{\mathrm{p}}\right)$. The potentials for current onset and for passivation are more positive for (111) electrodes than for (100) electrodes; this holds for both $\mathrm{n}$ - and p-type samples. The current density (or corresponding charge density) required to achieve passivation of the (100) surfaces is markedly larger than that for (111) electrodes. ${ }^{6,7}$

One of the problems associated with the study of (111) electrodes is the difficulty of preparing reproducibly defectfree surfaces. Macroscopic wafers usually have a misorientation that reveals steps; ${ }^{2,17,18}$ these may mask etching and electrochemical processes of the (111) surface. In 1987, Smith and co-workers presented an elegant approach to the preparation of macroscopic (111) surfaces. ${ }^{7}$ Anisotropic etching of $\mathrm{Si}(100)$ through square windows in an oxide mask gave inverted pyramids defined by (111) facets. Using such surfaces Smith and co-workers discovered rather interesting differences between (100) and (111) electrodes in $40 \% \mathrm{KOH}$ solution at $60^{\circ} \mathrm{C}$. In the (111) case the voltammogram was shown to depend on the potential scan rate for both $\mathrm{n}$ - and p-type electrodes. ${ }^{7}$ In subsequent work $^{19}$ chronoamperometric measurements were performed on the same well-defined (111) electrodes. In their papers, Smith et al. do not consider the charge carriers responsible for current (they mention that holes are required for anodic oxide formation). The chemistry underlying the anodic processes was also not discussed.

In the present study we reconsider the electrochemical oxidation of silicon. For our measurements we have, like Smith et al. ${ }^{7,19}$ and Kretschmer et al., ${ }^{8}$ used n- and p-type (100) wafers masked to reveal (111) facets during anisotropic chemical etching in $\mathrm{KOH}$ solution. In this way we could study the electrochemistry starting with the original (100) surface and 


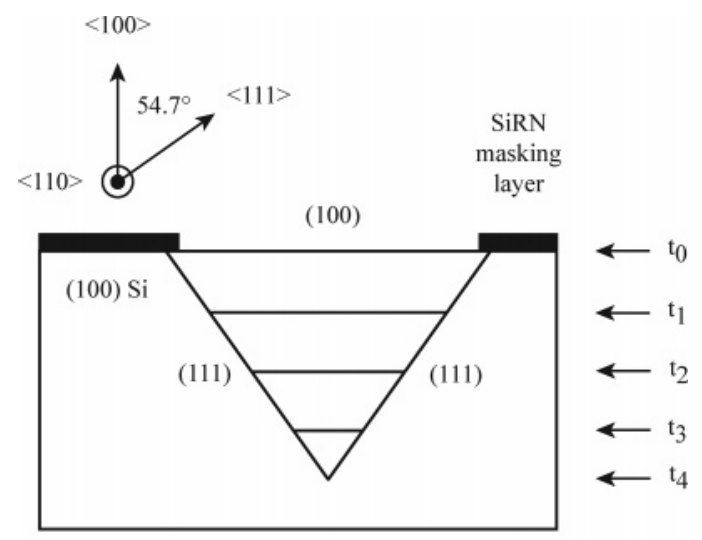

( a )

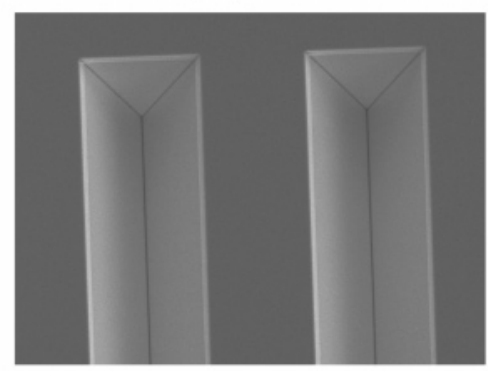

( b )

Figure 1. (a) Schematic representation of V-groove formation. At the start of the experiment $\left(t_{0}\right)$, only (100) faces are exposed to the etchant. After the grooves are fully formed $\left(t_{4}\right)$, the electrode consists of (111) faces. The relevant crystallographic directions are indicated by vectors. (b) SEM micrograph showing the end of the masked rectangular window, with fully formed $100 \mu \mathrm{m}$ wide $\mathrm{V}$-grooves.

follow the evolution of the $\mathrm{V}$-shaped grooves defined by (111) facets, finally obtaining pure (111) surfaces. ${ }^{2,8}$ This approach has the extra advantage of allowing a comparison of (100) and (111) orientations in the same wafer. Potentiodynamic and potential-step measurements were performed. The results allow us to draw conclusions about the mechanism and anisotropy of anodic dissolution and passivation.

\section{Experimental Section}

The starting point of our experiments was a 4-in. (100)oriented silicon wafer. Czochralski-grown n-type (phosphorus doped, $1-10 \Omega \cdot \mathrm{cm}$ ) and p-type (boron doped, 5-10 $\Omega \cdot \mathrm{cm}$ ) wafers were supplied by Okmetic. Well-defined (111) faces were formed by making an aligned mask pattern on the surface and etching the silicon anisotropically in $5.0 \mathrm{M} \mathrm{KOH}$ through the windows of the mask. Because the chemical etch rate of the (111) face of silicon is much lower than that of other faces, ${ }^{1,2}$ a V-shaped groove is formed, revealing (111) side walls (see Figure 1). With this approach we start with a (100) surface and end, when the groove is fully formed, with a (111) surface.

Two masking steps were used. A $300 \mathrm{~nm}$ thick silicon-rich nitride (SiRN) layer was deposited on the wafer by LPCVD (200 mTorr, $850{ }^{\circ} \mathrm{C}, 70 \mathrm{sccm} \mathrm{SiH}_{2} \mathrm{Cl}_{2}, 18 \mathrm{sccm} \mathrm{NH}$ ). Windows were opened in the nitride by a plasma etch (DRIE) followed by a phosphoric acid $(85 \%)$ etch. The first masking step with a "Vangbo mask" ${ }^{20}$ served to determine the $\langle 110\rangle$ crystallographic direction. After wet anisotropic etching, the pattern was inspected by optical microscopy. A second mask containing rectangular windows was then aligned with the edges along the $\langle 110\rangle$ direction with an accuracy of $0.05^{\circ}$ (see Figure 1). This pattern was used subsequently for etching $\mathrm{V}$-grooves. The masked wafers were diced into samples of $20 \times 20 \mathrm{~mm}^{2}$. To ensure a significant current in the electrochemical measurements, each sample contained an array of grooves. Two types of electrode were prepared. The A samples, used for measurements on (111) surfaces, had 25 grooves $(150 \mu \mathrm{m}$ wide; $8 \mathrm{~mm}$ long; interspacing, $150 \mu \mathrm{m}$; total (100) surface exposed to solution, $30.0 \mathrm{~mm}^{2}$ ). The B samples, used for measurements during groove formation, contained 30 grooves $(100 \mu \mathrm{m}$ wide; $8 \mathrm{~mm}$ long; interspacing, $100 \mu \mathrm{m}$; total (100) surface exposed to solution, $24.0 \mathrm{~mm}^{2}$ ). Before inspection of the etched grooves with optical microscopy the electrodes were rinsed thoroughly with deionized water (Millipore, $18 \mathrm{M} \Omega \cdot \mathrm{cm}$ ) and a $10 \%$ sulfuric acid solution (prepared from Merck \#1.00731, p.a. quality, 15min immersion). After these "quenching" steps the samples were rinsed again with deionized water and blown dry in a stream of argon.

On the basis of underetch at the mask edge, as determined by optical microscopy and the geometry of the system (see Figure 1), the (111) surface area could be calculated for the fully formed grooves. This was 55 and $40 \mathrm{~mm}^{2}$ for the A and B electrodes, respectively. For experiments on the (100) face, unmasked diced samples were used with a surface area of 39 $\mathrm{mm}^{2}$. All samples were provided with an Ohmic contact on the backside and mounted on a copper block in a Kel-F holder by means of a Viton O-ring. The silicon was the working electrode in a double-walled electrochemical cell with a platinum counter electrode and a saturated calomel electrode (SCE) as reference. All potentials are given with respect to SCE. A potentiostat (EG\&G Princeton Applied Research, PAR-273-A) was computercontrolled by LabView. A temperature sensor (Pt-100) and a thermostatic water bath (Haake Fisons F3 and S) were used to control the temperature in the double-walled cell (accuracy $\left.0.1{ }^{\circ} \mathrm{C}\right)$.

Before use, the samples were cleaned in concentrated nitric acid (65\%, Merck \#1.00456, p.a. quality, 30 min. immersion) to remove organic deposits and immersed for $15 \mathrm{~min}$ in deionized water. Native oxide was removed by a 1-min dip in a $1 \% \mathrm{HF}$ solution (Aldrich, p.a. quality). The etchant $(5.0 \mathrm{M}$ $\mathrm{KOH}$ ) was prepared from p.a. quality $\mathrm{KOH}$ (Merck \#1.05033, used as received) and deionized water and was continuously purged with argon during all measurements and pre-etches to remove dissolved gases.

The measurements were performed in the dark, unless otherwise stated. For experiments in which the electrode was illuminated, a halogen lamp (150 W, Schott KL-1500-E) was connected to a light-guiding fiber (diameter $5 \mathrm{~mm}$ ) that was inserted in a glass tube and positioned about $1 \mathrm{~cm}$ above the electrode surface. The illumination intensity was varied using neutral density filters and different settings of the light source.

\section{Results}

(1) Voltammetry during V-Groove Formation. The anisotropy in the electrochemistry of silicon becomes immediately obvious from the voltammograms as soon as groove-etching starts. Parts $\mathrm{a}$ and $\mathrm{b}$ of Figure 2 show results for $\mathrm{p}$ - and n-type silicon, respectively, measured at a constant scan rate toward positive potential. In both cases, the initial scan prior to anisotropic etching shows a single peak at lower potential, characteristic of the (100) surface..$^{7,21,22}$ The anodic current is due to oxidation of the silicon; below the peak potential the semiconductor dissolves, while passivation occurs at higher potential. Subsequently, voltammograms were measured at regular intervals during anisotropic etching. In both cases the (100) peak decreases and a second peak develops at considerably more positive potential as the (111) side walls are exposed. 

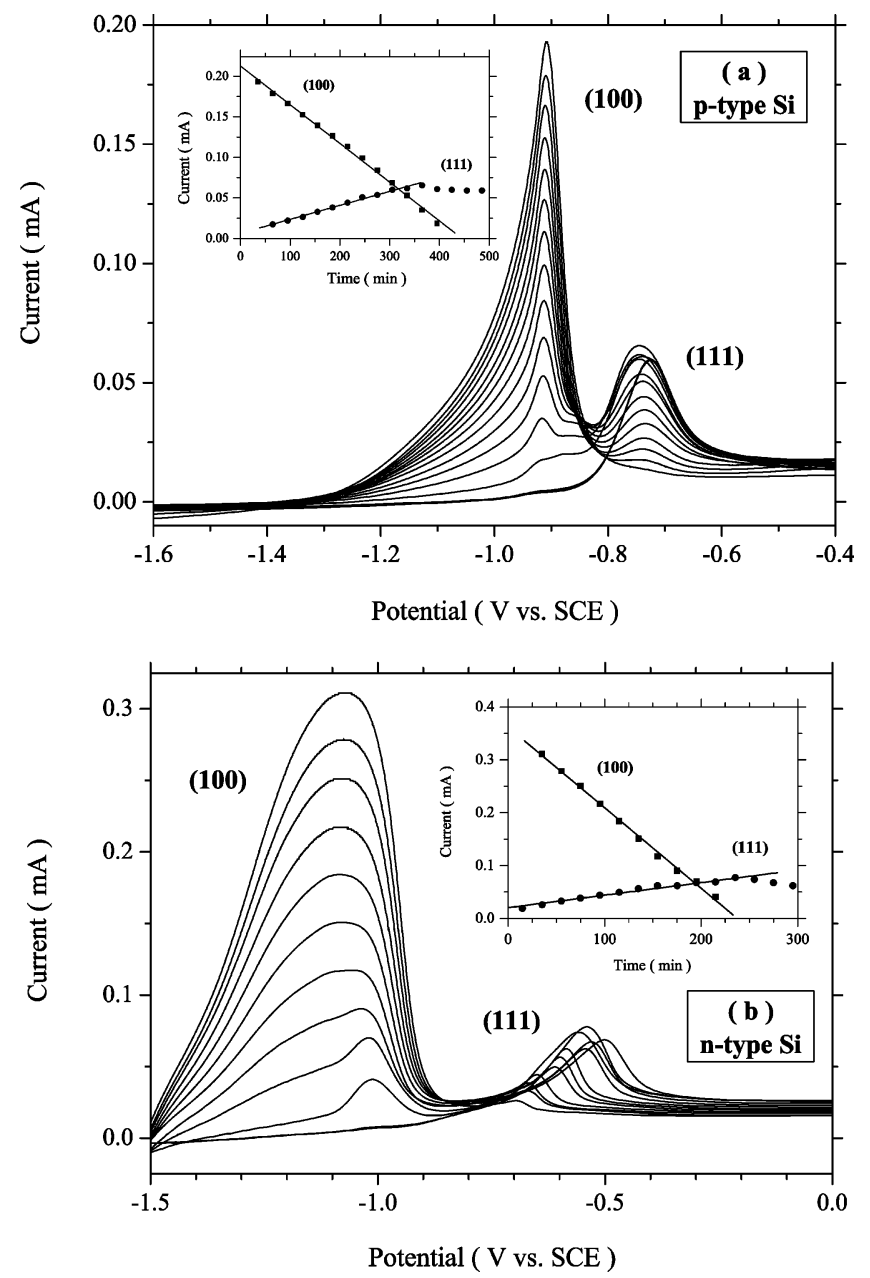

Figure 2. Voltammograms measured during V-groove formation. Groove width is $100 \mu \mathrm{m}$ and scan rate $10 \mathrm{mV} / \mathrm{s}$. The insets show the peak current for the (100) and (111) peaks as a function of time: (a) p-type $\mathrm{Si}, 5.0 \mathrm{M} \mathrm{KOH}, 50.0^{\circ} \mathrm{C}$, measured every $30 \mathrm{~min}$; (b) n-type $\mathrm{Si}$, $5.0 \mathrm{M} \mathrm{KOH}, 60.0^{\circ} \mathrm{C}$, measured every $20 \mathrm{~min}$.

Finally, when the groove is fully formed, only the peak corresponding to the (111) surface remains. ${ }^{7}$ What we are seeing is a gradual transition from a (100) to a (111) surface. In the inset of Figure 2a,b the peak current is shown as a function of etching time; in both cases we see a linear decrease for the (100) peak and a linear increase for the (111) peak. This is due to the constant rate of change of the area of the two faces, as would be expected for a wafer with this geometry, etching at a constant rate (see Figure 1). The (100) peak current goes to zero and the (111) peak levels off as the V-groove becomes fully formed. The figure shows the measured current and not the current density; the surface area of each crystal plane changes continuously during groove formation. In results reported by Kretschmer et al., ${ }^{8}$ comparable features are observed in the voltammograms of the n-type electrode. In their work there is an overlap of the (100) and (111) peaks, probably due to the higher temperature.

From our results it is clear that under these conditions the onset of anodic oxidation of silicon occurs at a more positive potential for the (111) electrode. This also holds for passivation, the sudden drop in current beyond the maximum. The difference in the peak potential of the two surfaces is more pronounced for the n-type electrode. During formation of the groove, the potential corresponding to the current maximum of the (100) and (111) surfaces of the p-type electrode does not change significantly as a function of time. This is also the case for the n-type (100) face (Figure 2b), except toward the end of groove formation. On the other hand, the peak potential of the n-type (111) face shifts continuously to positive potential for each subsequent scan (the total shift is about $200 \mathrm{mV}$ ).

(2) Influence of Scan Rate. The voltammograms shown in Figure 2 were recorded at a fixed scan rate of $10 \mathrm{mV} / \mathrm{s}$. Figure 3 shows the influence of scan rate. What is striking in this figure is the difference between (100) and (111) surfaces. As in Figure 2 , the onset of anodic oxidation and subsequent passivation of both (111) electrodes occurs at more positive potential than that of the corresponding (100) electrodes at all scan rates. For scan rates up to $100 \mathrm{mV} / \mathrm{s}$ and potentials negative with respect to the peak maximum the current-potential curves for both n-type and p-type (100) electrodes are independent of the scan rate (Figure 3a,c). At the highest scan rates $(50$ and $100 \mathrm{mV} / \mathrm{s}$ ) the peak current is raised somewhat and the peak is broadened; the peak potential shifts slightly to more positive values.

In contrast to the (100) electrodes, the voltammograms of the (111) electrodes respond strongly to scan rate. In the p-type (111) case (Figure 3b) the onset of the current shifts considerably to positive potential as the scan rate is increased. This shift is accompanied by a marked increase in the maximum current. The displacement of the voltammogram to positive potentials with increasing scan rate is even more spectacular for n-type Si(111) (Figure 3d). Another striking feature of Figure 3 is the magnitude of the anodic current. The peak current density of the (100) electrodes is an order of magnitude higher than that of the (111) electrodes at lower scan rates. ${ }^{7}$

Figure 4 shows a plot of the peak potential $\left(U_{\mathrm{p}}\right)$ for the two (111) electrodes as a function of scan rate. While for p-type silicon $U_{\mathrm{p}}$ tends to level off at higher scan rate (see also the inset of Figure 4), for n-type silicon $U_{\mathrm{p}}$ continues to increase up to the highest scan rate. (In the range $0.1-75 \mathrm{mV} / \mathrm{s}$, a displacement of more than $3 \mathrm{~V}$ is observed!) The strong scan rate dependence of the voltammograms of n- and p-type Si(111) electrodes indicates that kinetic effects are limiting the anodic current. The almost linear dependence of the peak potential on scan rate in the case of the n-type (111) surface suggests that potential is not important but that time is, in fact, the determining factor (see next section). Extrapolation to zero scan rate shows the same peak potential for $n$ - and p-type electrodes $(-0.871$ $\pm 0.003 \mathrm{~V}$; see the inset of Figure 4), in agreement with the results reported by Smith et al. ${ }^{7}$

(3) Current Transients for Anodization. If the scan rate dependence of the peak potential of n-type $\operatorname{Si}(111)$, shown in Figure 4, is primarily a temporal effect, then the time constant is approximately $50 \mathrm{~s}$ (the slope of a plot of $U_{\mathrm{p}}$ versus scan rate). The slow activation of the electrode should be revealed by measuring the current resulting from a potential step from the open-circuit value $\left(U_{\mathrm{oc}}\right)$ to a potential in the passive range. Results of such measurements for different temperatures are shown in Figure 5, in which the current density is plotted as a function of time following an abrupt change of the potential from open-circuit to $0.00 \mathrm{~V}$. All transients show a gradual increase to a maximum and a subsequent decrease; the latter is due to passivation of the electrode. Despite this strongly positive potential, the initial current is low at all temperatures. This slow activation of the current is strongly influenced by temperature. The time to reach maximum current decreases from almost $80 \mathrm{~s}$ at $40{ }^{\circ} \mathrm{C}$ to $8 \mathrm{~s}$ at $70{ }^{\circ} \mathrm{C}$ (see the inset of Figure 5).

That the process involved in current rise and decay in Figure 5 is indeed an oxidation and passivation of silicon that is initially retarded by the absence of charge carriers could be shown by irradiating the electrode after the potential was stepped. Curve a of Figure $6 \mathrm{a}$ is a transient measured in the 

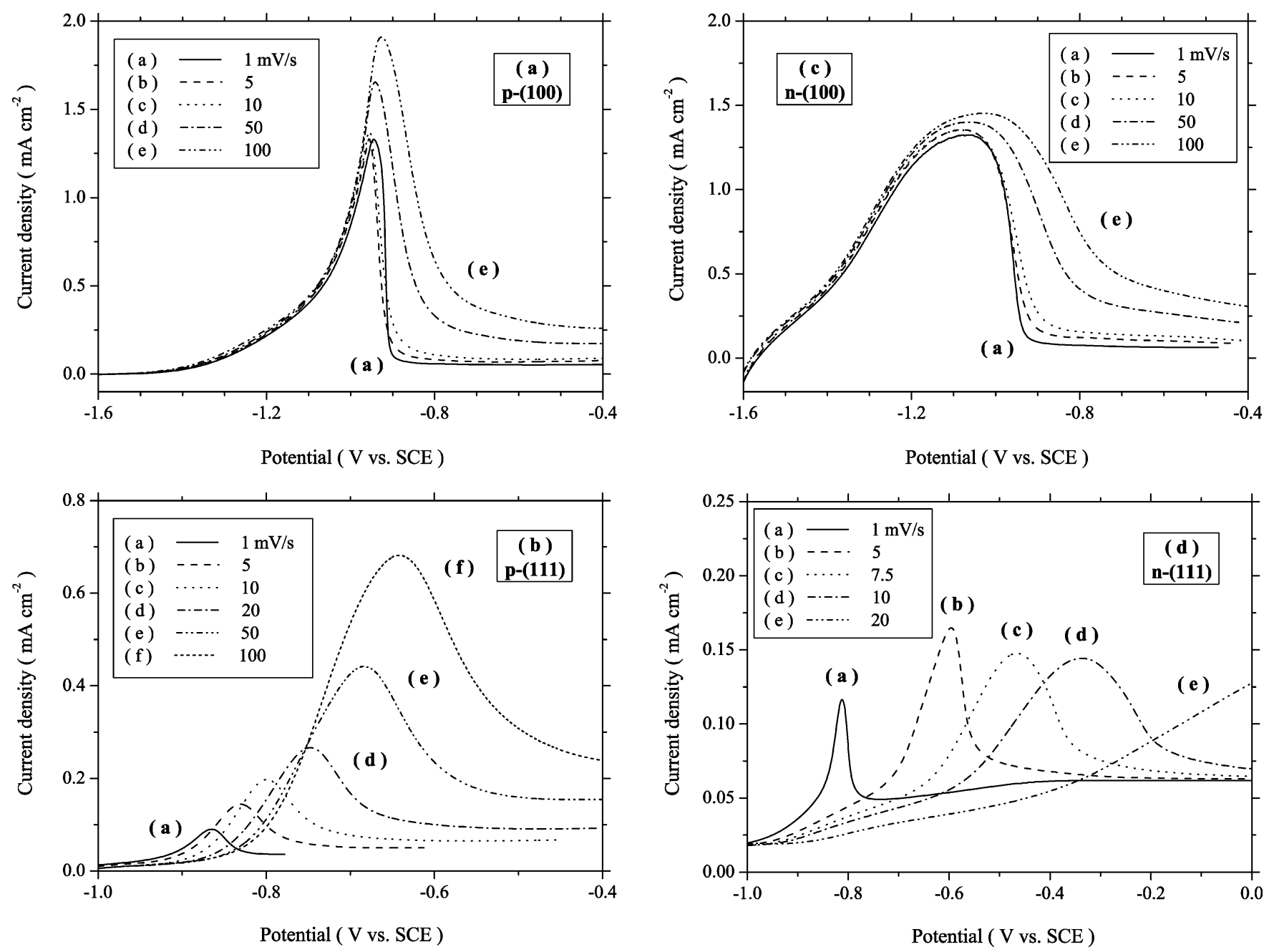

Figure 3. Voltammograms measured at various scan rates in $5.0 \mathrm{M} \mathrm{KOH}$ at $60{ }^{\circ} \mathrm{C}$ : (a) p-(100), (b) p-(111), (c) n-(100), (d) n-(111).

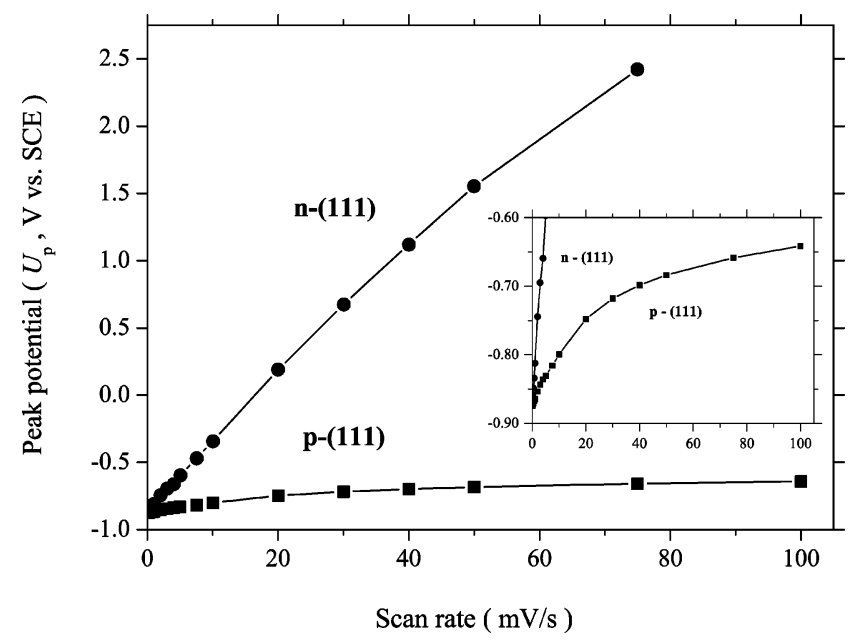

Figure 4. Peak potential $\left(U_{\mathrm{p}}\right)$ of $\mathrm{n}$ - and p-type $\mathrm{Si}(111)$ as a function of scan rate in $5.0 \mathrm{M} \mathrm{KOH}$ at $60{ }^{\circ} \mathrm{C}$. The inset shows a magnification for $\mathrm{p}-(111)$.

dark. For curves $b-e$, the electrode was subjected to a short pulse of white light at various stages during the transient measurement. Light gives rise to an anodic photocurrent $\left(I_{\text {photo }}\right)$ that is considerably higher than the transient dark current (note the logarithmic current scale). Electron-hole pairs, generated by light, are separated by the space-charge layer of the electrode. The holes arriving at the surface cause oxidation of the semiconductor. The decrease in photocurrent during the pulse

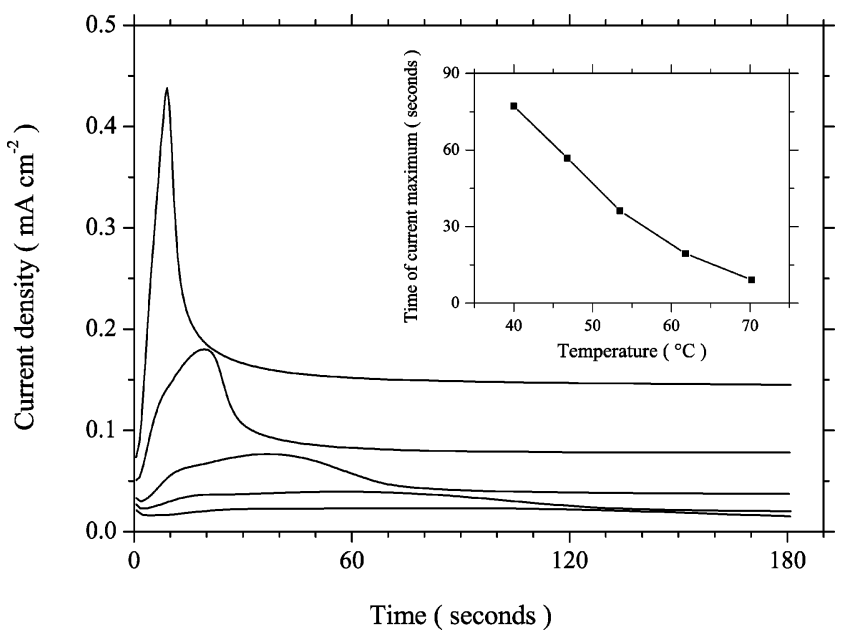

Figure 5. Current transients at various temperatures for n-type Si(111) after a potential step from $U_{\mathrm{oc}}$ to $0.00 \mathrm{~V}$. In the inset, the time to reach maximum current is shown as a function of temperature for the same experiments.

is due to passivation. When the light is switched off, the current drops abruptly and then slowly relaxes to a steady-state level. In all cases the photocurrent "kills" the dark current transient. The charge density under the transients measured in the dark (corrected for the background current) is about $2.4 \mathrm{mC} / \mathrm{cm}^{2}{ }^{7}$ If dissolution can be neglected, this charge density corresponds to four monolayers of oxidized silicon or a film thickness of 

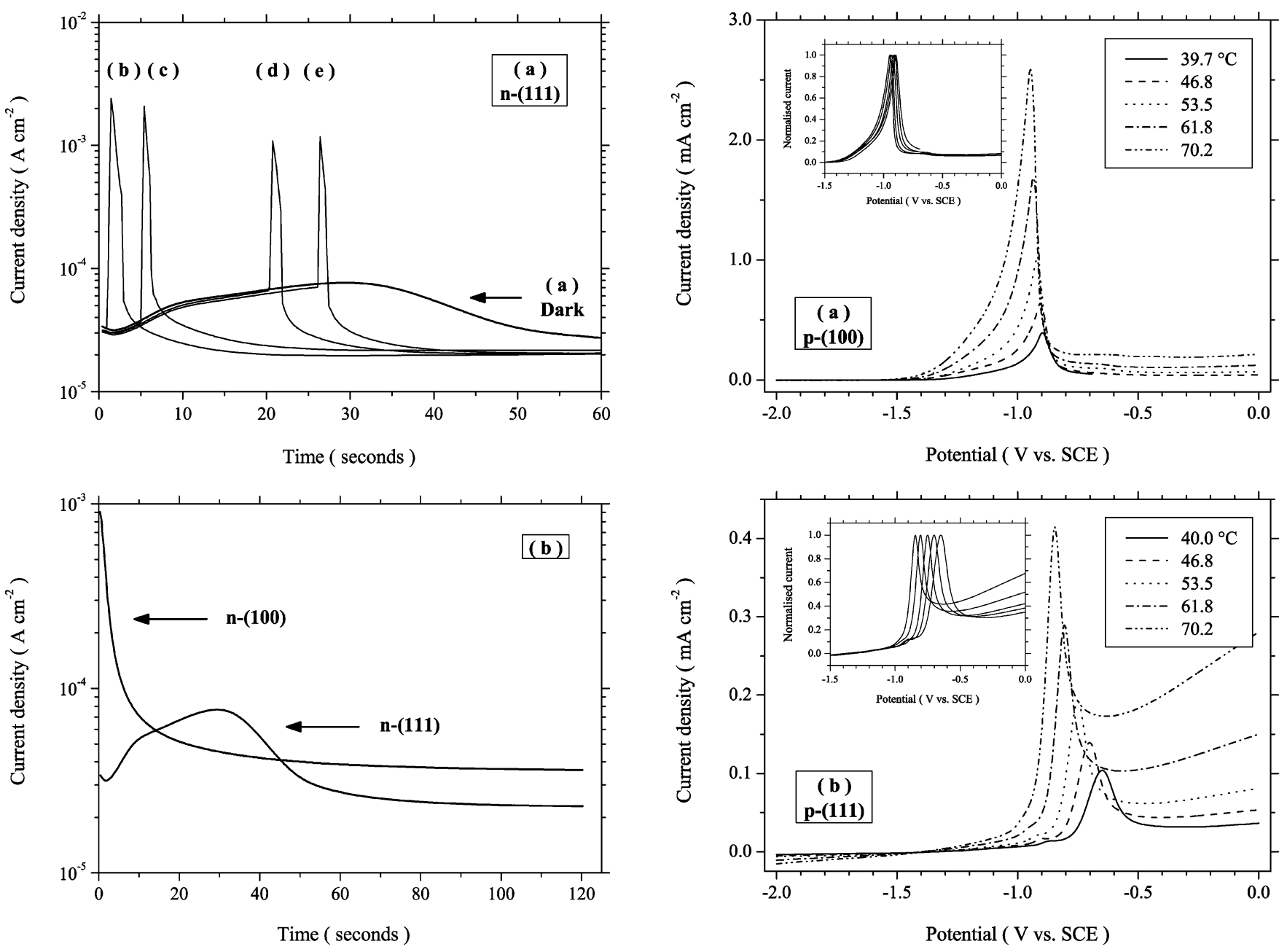

Figure 6. (a) Current transients following a potential step from $U_{\text {oc }}$ to $0.00 \mathrm{~V}$ for n-type $\mathrm{Si}(111)$ in $5.0 \mathrm{M} \mathrm{KOH}$ at $55.0^{\circ} \mathrm{C}$. The plot shows one transient measured in the dark (curve a) and transients for which the sample was subjected to a short pulse of light (curves b-e). (b) Current transients for n-type $\mathrm{Si}(100)$ and $\mathrm{Si}(111)$ measured in the dark. Conditions are identical to those used in part a. Note the logarithmic current scales of both figures.

$20 \AA$ (the density of anodic oxide is expected to be lower than that of thermal oxide ${ }^{23}$ ). The charge in the photocurrent pulse needed to suppress the dark current is comparable to that under the dark current transient. This value also agrees quite well with the charge under the anodic peak of the voltammograms for the (111) electrodes measured at higher scan rates.

In Figure $6 \mathrm{~b}$ a current transient for an n-type (100) electrode is shown for comparison with the corresponding measurement on the (111) electrode. Clearly, no induction of the anodic process is observed in the (100) case after the potential is stepped; an initial high current is followed by a monotonic decrease to a steady-state value. Similar transients are also found with p-type (100) electrodes. Although measurement conditions differ, the transients measured for (100) electrodes, both n- as well as p-type, correspond well to those of Raisch et al. ${ }^{14,24}$

(4) Influence of Temperature on Voltammograms. In the case of p-type (100) and (111) electrodes, a change in temperature does not alter the basic shape of the voltammogram measured at a fixed scan rate (Figure 7a,b). An increase in temperature increases the current maximum of both electrodes considerably. At all temperatures, the current density corresponding to the maximum of the (100) electrode is significantly higher than that of the (111) electrodes. Additionally, for both (111) electrodes there is a marked shift of the peak in the

Figure 7. Voltammograms measured with (a) p-type $\mathrm{Si}(100)$ and (b) $\mathrm{Si}(111)$ as a function of temperature in $5.0 \mathrm{M} \mathrm{KOH}$ at a scan rate of $10 \mathrm{mV} / \mathrm{s}$. The insets show current-potential curves with current normalized with respect to the peak current.

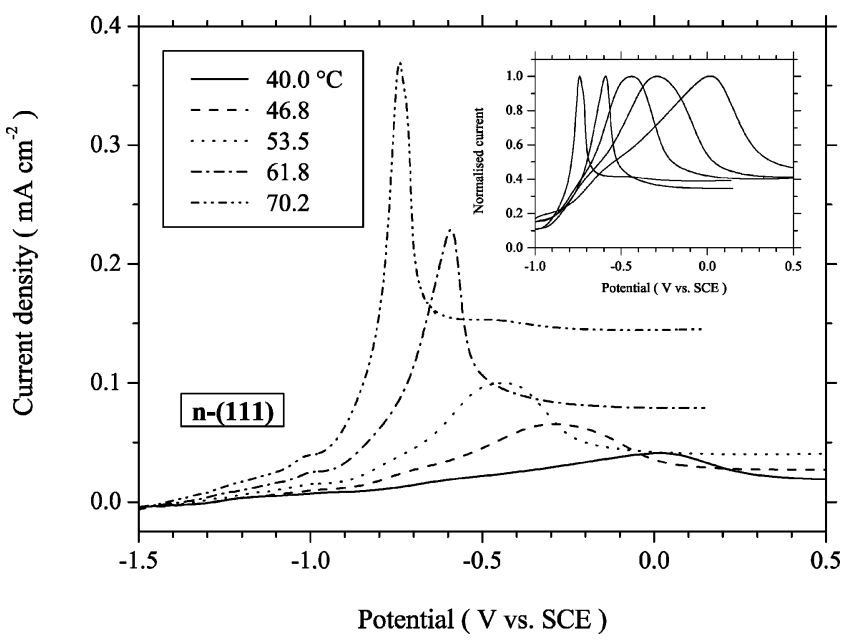

Figure 8. Voltammograms of n-type $\mathrm{Si}(111)$ measured as a function of temperature in $5.0 \mathrm{M} \mathrm{KOH}$ solution at a scan rate of $10 \mathrm{mV} / \mathrm{s}$. The inset shows current-potential curves with current normalized with respect to the peak current.

voltammogram to negative potentials. This is particularly clear in the insets showing normalized current-potential curves. The effect on the voltammograms of the n-type (111) electrode (Figure 8) is most striking. The very broad, low current peak measured at the lowest temperature is raised and the peak current 
increases. The peak shifts by almost $750 \mathrm{mV}$ to negative potential for a temperature increase of just $30{ }^{\circ} \mathrm{C}$ (see the inset of Figure 8). The strong temperature dependence seen in Figure 8 is perhaps not surprising when we consider the effect of temperature on the induction time observed in the potentialstep measurements (see Figure 5). Both results suggest the important role of a chemical activation of the electrochemical reaction.

\section{Discussion}

There are three important issues to be considered in relation to the present electrochemical results: (i) the nature of the anodic reaction (dissolution or oxide formation), (ii) the mechanism of the reaction (a valence or conduction band process), and (iii) the role of the crystallographic orientation.

Because of the reported coupling of chemical and electrochemical steps, ${ }^{12}$ it is useful first to reconsider briefly the chemistry of the anisotropic etching reaction at open-circuit potential $\left(U_{\text {oc }}\right)$.

(1) Anisotropic Etching. Chemical etching of silicon can be represented in a simplified mechanism by two reactions: ${ }^{10,13}$

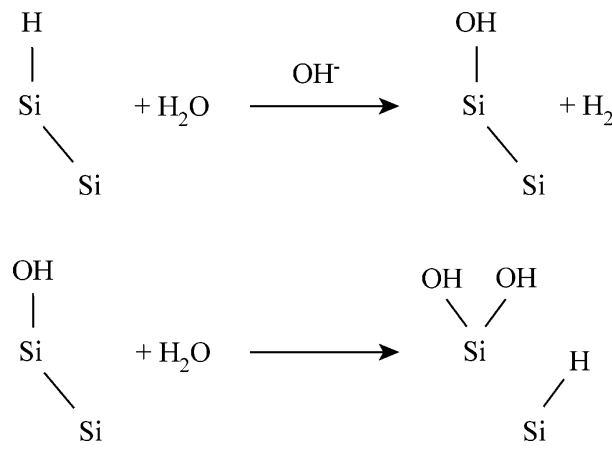

The essential step is the breaking of the $\mathrm{Si}-\mathrm{Si}$ back-bond by a water molecule (eq 2). This reaction yields an $\mathrm{Si}-\mathrm{OH}$ surface bond and an $\mathrm{Si}-\mathrm{H}$ bond on the subsurface silicon atom. When the surface silicon atom dissolves, ${ }^{25}$ after its remaining backbonds are broken, an $\mathrm{Si}-\mathrm{H}$ species is exposed to solution that is subsequently replaced by $\mathrm{Si}-\mathrm{OH}$ via an $\mathrm{OH}^{-}$-catalyzed reaction (eq 1). ${ }^{13,21,26-29}$ In contrast to the "covalent" $\mathrm{Si}-\mathrm{H}$ bond, the $\mathrm{Si}-\mathrm{OH}$ is quite polar; ${ }^{30,31}$ the $\mathrm{OH}$ group polarizes the $\mathrm{Si}-\mathrm{Si}$ back-bond, making it susceptible to attack by water in reaction $2 .{ }^{31,32}$ Whether the silicon surface is mainly $\mathrm{OH}-$ or H-terminated during anisotropic etching will depend on the relative rates of reactions 1 and 2 .

The marked difference in etching properties of (100) and (111) surfaces can be related to differences in the chemical reactivity of the two surfaces. Two factors are important. The first is the stability of the $\mathrm{Si}-\mathrm{H}$ bond, which determines the rate of formation of $\mathrm{Si}-\mathrm{OH}$ (reaction 1). In the literature there is agreement that the monohydride present on the (111) surface is chemically considerably more stable than the dihydride on the (100) surface..$^{23,31,34}$ The second factor is that the ease with which a silicon atom can be removed depends to a large extent on the number of back-bonds [three in the case of (111) and two in the case of (100) surfaces]. These factors can account for the much higher etch rate of $\mathrm{Si}(100)$ and the observation that terraces on (111) surfaces are "stable" and that etching of $\mathrm{Si}(111)$ follows a step-flow mechanism. ${ }^{1,2,17,18}$

(2) Anodic Oxidation: n-Type Si. Anodic oxidation of an n-type semiconductor can only occur by injection of electrons from surface bonds into the conduction band. Since direct excitation of a bonding electron from the valence to the
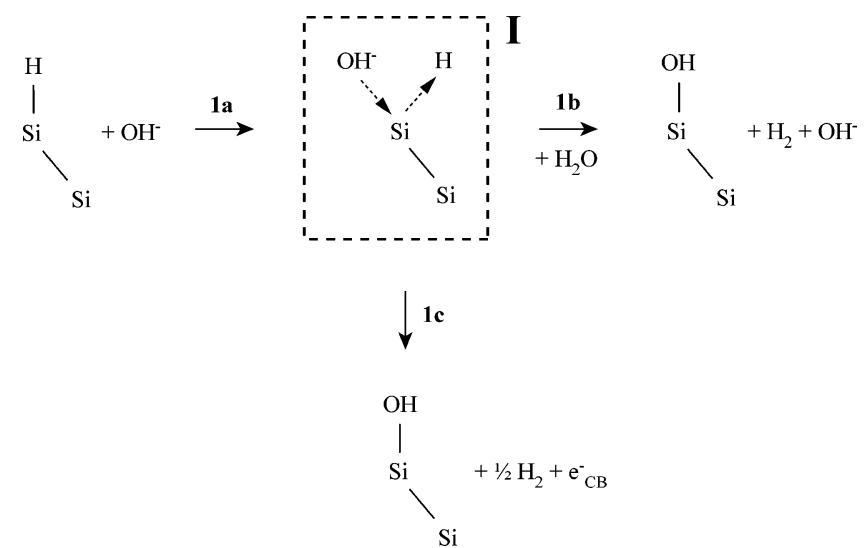

Figure 9. Reaction scheme 1, showing the first step of the reaction mechanism. For clarity, only one surface and subsurface silicon atom are shown. The intermediate is framed and denoted by $\mathbf{I}$.
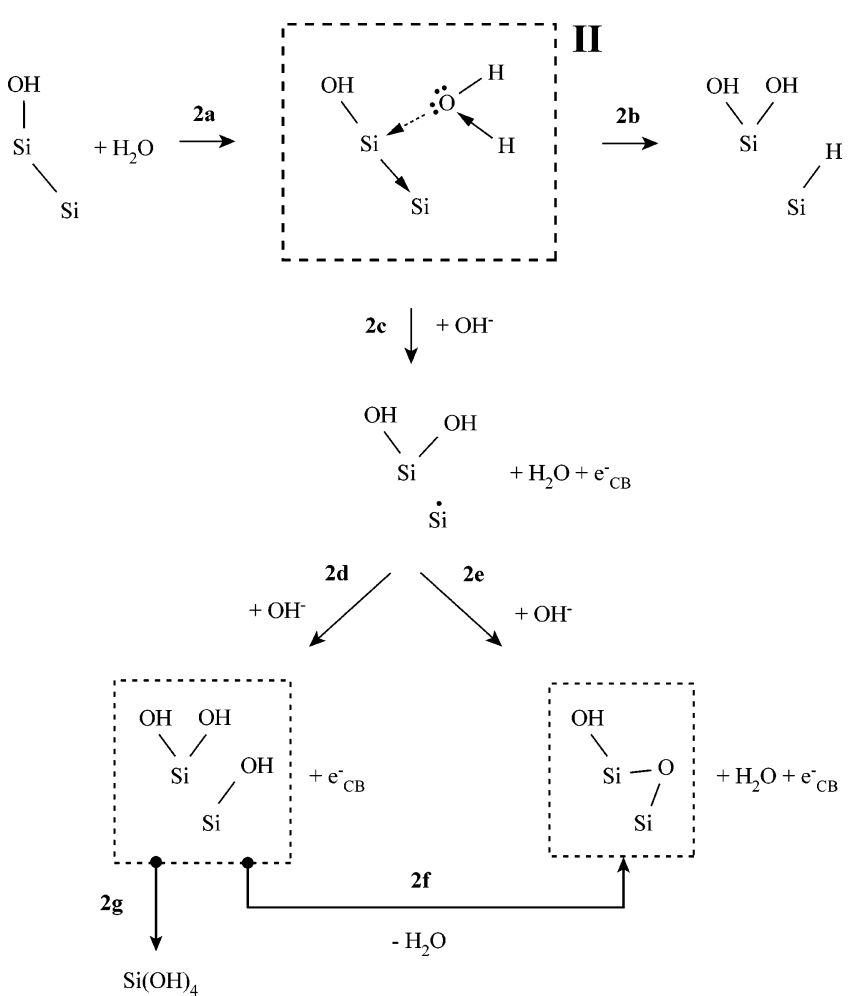

Figure 10. Reaction scheme 2, showing the second step of the reaction mechanism. The intermediate is framed and denoted by II.

conduction band of silicon is energetically unfavorable, the large anodic current observed with the n-type (100) electrode in $\mathrm{KOH}$ solution (see Figure 3c) must be due to states with an energy level high in the band gap. Previous work ${ }^{12}$ has indicated that the states responsible are intermediates of the chemical etching reaction. Reactions 1 and 2 are considered in more detail in the two general reaction schemes of Figures 9 and 10; the intermediates are denoted by I and II in these schemes. Intermediate I results from nucleophilic attack by an $\mathrm{OH}^{-}$ion on the $\mathrm{Si}-\mathrm{H}$ surface bond (step 1a, Figure 9); the "departing hydride" reacts with water to give hydrogen gas and an $\mathrm{OH}^{-}$ ion (step 1b). ${ }^{27}$ Intermediate II very likely results from the interaction of an electron lone pair on the oxygen of a water molecule with the surface silicon atom positively polarized by the $\mathrm{OH}$ group (step 2a, Figure 10). Step $2 \mathrm{~b}$ could be seen as a proton exchange between water and the subsurface silicon atom.

At potentials positive with respect to the open-circuit value, electrons are injected from these intermediates into the conduc- 
tion band and reach the back contact to give an anodic current in the external circuit. This implies a competition between a chemical and an electrochemical process in the two main reaction steps ( $1 \mathrm{~b}$ versus $1 \mathrm{c}$ and $2 \mathrm{~b}$ versus $2 \mathrm{c}$ ). Electron injection in the second step of the reaction mechanism (see Figure 10) very likely involves two consecutive one-electron steps. Both chemical and electrochemical reactions result in a fully oxidized silicon atom. However, as is clear from reaction scheme 2 (Figure 10), the final product of electrochemical oxidation may be a silicate species $\mathrm{Si}(\mathrm{OH})_{4}$ in solution (via step $2 \mathrm{~d}$, followed by step $2 \mathrm{~g}$ ) or a surface oxide (via step 2e). Oxide can also be formed by dehydration of two adjacent $\mathrm{Si}-\mathrm{OH}$ species (step 2f). ${ }^{31,32,35}$ The surface will passivate if the oxide coverage becomes high. Oxide also dissolves in a chemical reaction and this gives rise to a small steady-state current. Oxide dissolution is not shown in the scheme. Because of uncertainty with regard to the potential distribution at the semiconductor/solution interface in the active/passive range, ${ }^{10}$ this model can, at the moment, only be used qualitatively.

As mentioned previously, a much larger charge density is required for anodic passivation of (100) electrodes. This result might be due to a relatively higher rate constant for reaction $2 \mathrm{e}$ at (111) electrodes, possibly due to steric effects. ${ }^{27}$ Dehydration of $\mathrm{Si}-\mathrm{OH}$ bonds (reaction $2 \mathrm{f}$ ) to give oxide is more likely to occur at (111) surfaces. ${ }^{31,32,35}$ With two intact back-bonds, the surface silicon atom is more firmly anchored in the lattice, thus favoring dehydration and oxide formation (reaction $2 \mathrm{f}$ ); in the (100) case the surface atom with just one back-bond and an $\mathrm{Si}(\mathrm{OH})_{3}$ configuration is much more likely to dissolve (reaction $2 \mathrm{~g})$.

Since the chemical etching of $\mathrm{Si}(100)$ is a fast process, occurring "uniformly" over the whole surface, the intermediates I and II must be formed at a significant rate and with a uniform spatial distribution. If the rate constants for electron injection from the intermediates are sufficiently high, then a fast injection of electrons and a high anodic current can be expected. To account for the transient in Figure $6 \mathrm{~b}$ and the voltammograms the maximum current corresponding to steps $2 \mathrm{~d}$ and $2 \mathrm{e}$ must be larger than the peak current in the voltammogram, i.e., that needed to passivate the (100) surface. Since the peak current represents only a small fraction of the total chemical dissolution rate (a few percent), a kinetic limitation of electron injection is not expected.

For the n-type (111) surface the situation is different. Chemical etching occurs predominantly at step edges, which are much more reactive than the (111) terraces. ${ }^{17,18}$ The etch rate is consequently much lower than that of the (100) surface. Since, in electrochemical experiments the whole surface ultimately passivates, anodic oxidation must occur both at step edges and on the terraces. The former should be fast, as for the (100) surface; the latter should be slow because of the stability of the terrace. In a potential-step measurement on n-(111) facets, it is therefore not surprising that the anodic current is initially low (see Figures 5 and 6). The contribution of step edges to the current is small. For slightly misaligned (111) surfaces or (111) facets that have been electrochemically roughened, we find transients showing a high initial current with a fast decay (corresponding to steps etc.) followed by a slow rise to a maximum (as for the (111) facets). Voltammograms for such nonideal surfaces also deviate from the current-potential curves of the anisotropically etched (111) facets.

The slow activation of the (111) surface can be caused by different effects. If on the terraces reaction 1a is rate-limiting, then the rate constant $k_{1 \mathrm{a}}$ determines the initial total rate, while

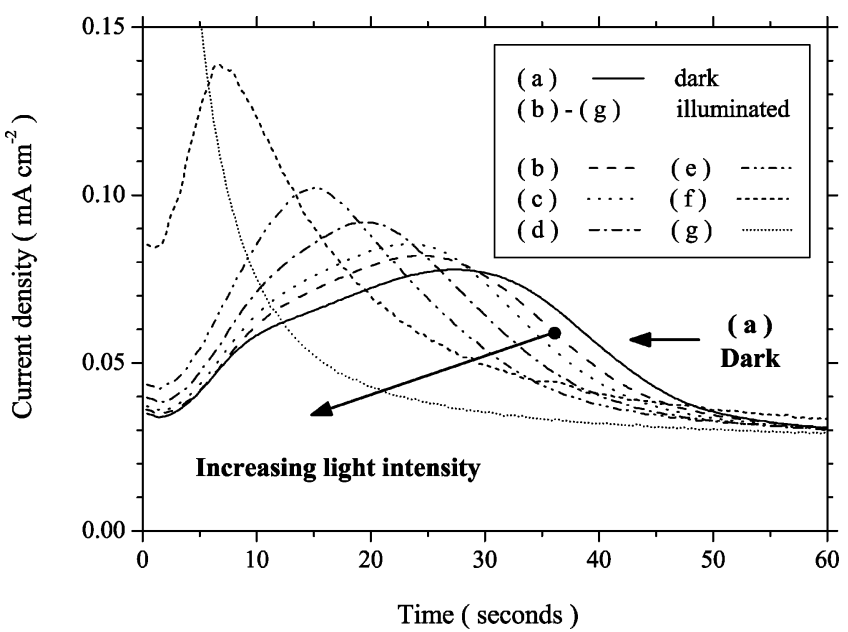

Figure 11. Current transients for n-type $\mathrm{Si}(111)$ in the dark (curve a) and under continuous illumination for various light intensities (increasing from curve b to $\mathrm{g}$ ) following a potential step from $U_{\mathrm{oc}}$ to $0.00 \mathrm{~V}$ in 5.0 $\mathrm{M} \mathrm{KOH}$ at $55.0^{\circ} \mathrm{C}$. The corresponding photocurrent densities are $1.2,2.2,4.8,8.6,50.3$, and $580 \mu \mathrm{A} / \mathrm{cm}^{2}$.

the ratios $k_{1 \mathrm{~b}} / k_{1 \mathrm{c}}$ and $k_{2 \mathrm{~b}} / k_{2 \mathrm{c}}$ decide the relative contributions of chemical etching and electron injection. It is important to note that the electrochemical path changes the surface chemistry and thus the relative contributions of the two processes. While the product resulting from both chemical and electrochemical reactions is a fully oxidized silicon atom, the way in which the product is formed is different for the two cases. Whereas the chemical step $2 b$ regenerates the hydride surface, the electrochemical step $2 \mathrm{c}$ produces either a hydroxylated surface $(2 \mathrm{c}+$ $2 \mathrm{~d})$ or an oxide $(2 \mathrm{c}+2 \mathrm{e})$. Reaction $2 \mathrm{c}$ thus replaces $\mathrm{Si}-\mathrm{H}$ termination of the subsurface silicon atom by $\mathrm{Si}-\mathrm{OH}(2 \mathrm{~d})$ or an oxygen bridge (2e). Reaction sequence $2 c+2 d$ forms a selfsustaining loop (the surface $\mathrm{Si}-\mathrm{OH}$ is replaced by a "subsurface" $\mathrm{Si}-\mathrm{OH})$. As this process develops the surface becomes $\mathrm{OH}$-terminated and current generation becomes independent of reaction scheme 1 (Figure 9). In a potential-step measurement the anodic current will increase (with a time constant determined by $k_{2 \mathrm{~b}} / k_{2 \mathrm{c}}$ ). For the case of $\mathrm{Si}(111)$, we have seen that oxide formation is more favorable than silicon dissolution. Reaction sequence $2 c+2 \mathrm{e}$ giving surface oxide will have an effect similar to that of the sequence $2 \mathrm{c}+2 \mathrm{~d}$. $\mathrm{A} \mathrm{Si}-\mathrm{Si}$ bond adjacent to a bridged oxygen will be polarized in the same way as with $\mathrm{Si}-\mathrm{OH}$. One bridged oxygen destabilizes electrochemically three $\mathrm{Si}-\mathrm{Si}$ bonds, giving an "autocatalytic" effect on the current. In addition, mechanical strain on the lattice as a result of oxygen incorporation will lead to destabilization of adjacent $\mathrm{Si}-\mathrm{Si}$ bonds. ${ }^{31,32}$ This effect should also accelerate oxidation and thus electron injection.

If, as suggested in the previous paragraph, the formation of oxide "nuclei" 35 is important for activation of the surface, then it should be possible to use light to accelerate the process. For the experiments in Figure 11, a low background of white light was used during potential-step measurements. As in the experiments of Figure 6a, illumination of n-type $\mathrm{Si}(111)$ in the passive range gives rise to a photocurrent leading to oxide formation. For the four lowest intensities in Figure 11 (curves b-e), the current under illumination at $t=0$ is the same as or only very slightly higher than the dark current (curve a). Although the photocurrent is very low, nevertheless illumination has a striking effect on the transient: activation of the surface is clearly enhanced. With increasing light intensity, a faster rise time of the transient is observed (compare with curve a, which shows the dark-current transient), and the time to reach the maximum 
becomes shorter, while the maximum current increases. It is important to realize that in these experiments the charge corresponding to the photocurrent is much smaller than that involved in the dark current transient (i.e. that needed to passivate the surface). For the second highest light intensity (curve f) with a photocurrent $\left(50.3 \mu \mathrm{A} / \mathrm{cm}^{2}\right)$ slightly larger than the initial dark current, the general shape of the transient is similar to that measured at lower intensity (and in the dark), but in this case activation is very fast (4 times faster than in the dark). At the highest light intensity (curve g, $I_{\text {photo }}=580 \mu \mathrm{A} /$ $\mathrm{cm}^{2}$ ), the electrode is photoanodically passivated as in the experiments of Figure 6a. The transient is similar to that of n-type $\operatorname{Si}(100)$ (see Figure 6b).

(3) Anodic Oxidation: p-Type Si. In a p-type electrode, direct oxidation of surface bonds by valence band holes can occur. However, as pointed out in the Introduction, there is considerable evidence to show that for potentials before passivation occurs the anodic current of p-type $\mathrm{Si}(100)$ is due, to a large extent, to electron injection. The dependence of the current-potential curve of p-type $\mathrm{Si}(111)$ on scan rate is considerable (see Figure $3 b$ ) and, like in the n-type case, this suggests a kinetic inhibition of the anodic process. Smith et al. report step experiments ${ }^{19}$ from $U_{\mathrm{oc}}$ to potentials between the peak maximum and the onset of the passive range. The form of the current transient with a slow rise to a maximum is similar to that for n-type $\mathrm{Si}(111)$ (Figure 5). We have reproduced their results with our electrodes. Again, this indicates a relatively slow chemical activation of the electrochemical process. This is further confirmed by the strong effect of temperature on the voltammogram, as seen in Figure $7 \mathrm{~b}$ (compare with the p-type (100) electrode in Figure 7a). These results suggest that in the peak range charge transfer occurs via an activated intermediate of the chemical reaction. Whether this involves electron injection from or hole trapping by the intermediate is not clear. Electrical impedance measurements do not give us dependable information about potential distribution and surface hole densities in the peak potential range. ${ }^{10}$

Hole reactions certainly play a role in the oxidation of p-type silicon at more positive potential. For example, for step experiments into the passive range the current transients for $n$ and p-type $\mathrm{Si}(111)$ differ considerably. In the n-type case, the general shape of the transient does not depend markedly on the final potential. On the other hand, transients measured with p-type $\operatorname{Si}(111)$ when the potential is stepped even to the onset of the passive range resemble those of $\mathrm{Si}(100) .{ }^{19}$ This must be due to the participation of holes in a direct anodic oxidation process. The hole contribution also explains why the oxide continues to grow as the potential is increased in the passive range. ${ }^{7,21}$ This is not possible for the oxidation of the n-type semiconductor in the dark, which depends on a chemical reaction between water and back-bonds; further oxide growth is, of course, possible under illumination when holes are photogenerated.

\section{Conclusions}

Striking differences are observed in the kinetics of anodic oxidation of $\mathrm{Si}(100)$ and $\mathrm{Si}(111)$ electrodes. The voltammograms of n- and p-type $\mathrm{Si}(100)$ in 5.0 M KOH solution depend only slightly on the potential scan rate up to $100 \mathrm{mV} / \mathrm{s}$. On the other hand, a marked dependence on scan rate is observed for Si(111), the effect being much stronger for the n-type material. It is concluded that the anodic current responsible for oxidation results from electron injection into the conduction band. The injecting species are reactive intermediates of chemical etching.
The slow kinetics of the electrochemical reaction at the (111) electrodes is therefore attributed to the chemical stability of the (111) surface. That chemical activation of the surface is a prerequisite for current flow is clear from current-transient measurements with n-type $\mathrm{Si}(111)$ following a step from opencircuit potential to a value in the passive range. Potentiodynamic and potential-step measurements at different temperatures and photocurrent measurements confirm this hypothesis. A schematic model involving coupled chemical and electrochemical reactions can account for this anisotropy in anodic oxidation of $\mathrm{Si}$. The model can also explain why considerably less charge is required to passivate the (111) electrode.

Acknowledgment. The authors thank Duy Nguyen, Henri Jansen $\left(\mathrm{MESA}^{+}\right.$Research Institute, University of Twente, The Netherlands), and Ismail Shah (Radboud University Nijmegen, The Netherlands) for valuable discussions and Erwin Berenschot and Meint de Boer $\left(\mathrm{MESA}^{+}\right.$Research Institute, University of Twente, The Netherlands) for their help with wafer processing. Arno Steckenborn (Siemens AG, Berlin, Germany) is acknowledged for his interest and support. This work was financially supported by the Dutch Technology Foundation (STW, TPC5990).

\section{References and Notes}

(1) Seidel, H.; Csepregi, L.; Heuberger, A.; Baumgärtel, H. J. Electrochem. Soc. 1990, 137, 3612.

(2) Elwenspoek, M.; Jansen, H. In Silicon Micromachining, Cambridge Studies in Semiconductor Physics and Microelectronic Engineering; Cambridge University Press: Cambridge, UK, 1998; Vol. 7.

(3) Oosterbroek, E.; van den Berg, A. In Lab-on-a-Chip-Miniaturized Systems for (Bio)Chemical Analysis and Synthesis; Elsevier: Amsterdam, 2003.

(4) Ashruf, C. M. A.; French, P. J.; Bressers, P. M. M. C.; Kelly, J. J.; Sarro, P. M. Sensors Actuators A 1998, 66, 284.

(5) Collins, S. D. J. Electrochem. Soc. 1997, 144, 2242.

(6) Glembocki, O. J.; Stahlbusch, R. E. J. Electrochem. Soc. 1985, $132,145$.

(7) Smith, R. L.; Kloeck, B.; Rooij, N. de; Collins, S. D. J. Electroanal. Chem. 1987, 238, 103.

(8) Kretschmer, H.-R.; Xia, X. H.; Kelly, J. J.; Steckenborn, A. J. Electrochem. Soc. 2004, 151, C633.

(9) D’Arrigo, G.; Spinella, C.; Rimini, E.; Rubino, L.; Lorenti, S. J. Electrochem. Soc. 2004, 151, C523.

(10) Xia, X. H.; Kelly, J. J. Phys. Chem. Chem. Phys. 2001, 3, 5304.

(11) (a) Gerischer, H.; Mindt, W. Electrochim. Acta 1968, 13, 1329. (b) Memming, R. In Semiconductor Electrochemistry; Wiley-VCH: Weinheim, 2001. (c) Notten, P. H. L.; van den Meerakker, J. E. A. M.; Kelly, J. J. In Etching of III-V Semiconductors-An Electrochemical Approach; Elsevier: Oxford, UK, 1991.

(12) Xia, X.; Ashruf, C. M. A.; French, P. J.; Rappich, J.; Kelly, J. J. J. Phys. Chem. B 2001, 105, 5722.

(13) Allongue, P.; Costa-Kieling, V.; Gerischer, H. J. Electrochem. Soc. 1993, 140, 1018.

(14) Raisch, P.; Haiss, W.; Nichols, R. J.; Schiffrin, D. J. J. Phys. Chem. B 2001, 105, 12508.

(15) Cattarin, S.; Peter, L. M.; Riley, D. J. J. Phys. Chem. B 1997, 101, 4071.

(16) (a) Schmidt, B.; Borany, J. von; Todt, U.; Erlebach, A. Sensors Actuators A 1994, 41-42, 689. (b) Huster, R.; Stoffel, A. Sensors Actuators A 1990, 21-23, 899 .

(17) Allongue, P.; Costa-Kieling, V.; Gerischer, H. J. Electrochem. Soc. 1993, $140,1009$.

(18) Allongue, P.; Brune, H.; Gerischer, H. Surf. Sci. 1992, 275, 414.

(19) Smith, R. L.; Kloeck, B.; Collins, S. D. J. Electrochem. Soc. 1988 135, 2001.

(20) Vangbo, M.; Bäcklund, Y. J. Micromech. Microeng. 1996, 6, 279.

(21) Bressers, P. M. M. C.; Pagano, S. A. S. P.; Kelly, J. J. J. Electroanal. Chem. 1995, 391, 159.

(22) Cattarin, S.; Musiani, M. M. J. Phys. Chem. B 1999, 103, 3162.

(23) Zhang, X. G. In Electrochemistry of Silicon and its Oxide; Kluwer Academic/Plenum Publishers: New York, 2001. 
(24) Haiss, W.; Raisch, P.; Schiffrin, D. J.; Bitsch, L.; Nichols, R. J. Faraday Discuss. 2002, 121, 167.

(25) Nijdam, A. J.; van Veenendaal, E.; Gardeniers, J. G. E.; Kentgens, A. P. M.; Nachtegaal, G. H.; Elwenspoek, M. J. Electrochem. Soc. 2000 147, 2195.

(26) Baum, T.; Schiffrin, D. J. J. Electroanal. Chem. 1997, 436, 239.

(27) Baum, T.; Schiffrin, D. J. J. Chem. Soc., Faraday Trans. 1998, 94, 691.

(28) Wind, R. A.; Hines, M. A. Surf. Sci. 2000, 460, 21

(29) Wind, R. A.; Jones, H.; Little, M. J.; Hines, M. A. J. Phys. Chem. B 2002, 106, 1557.

(30) Allongue, P. Phys. Rev. Lett. 1996, 77, 1986.
(31) Gräf, D.; Grundner, M.; Schulz, R. J. Vac. Sci. Technol. A 1989 7, 808.

(32) Boonekamp, E. P.; Kelly, J. J.; van de Ven, J.; Sondag, A. H. M J. Appl. Phys. 1994, 75, 8121.

(33) Rappich, J.; Lewerenz, H. J.; Gerischer, H. J. Electrochem. Soc. 1993, 140, L187.

(34) (a) Pietsch, P. J.; Chabal, Y. J.; Higashi, G. S. Surf. Sci. 1995, 331-333, 395. (b) Dumas, P.; Chabal, Y. J.; Jakob, P. Surf. Sci. 1992 $269-270,867$.

(35) Kasparian, J.; Elwenspoek, M.; Allongue, P. Surf. Sci. 1997, 388, 50. 